\title{
Different Properties of Visual Relearning after Damage to Early Versus Higher-Level Visual Cortical Areas
}

\author{
Anasuya Das, Margaret DeMagistris, and Krystel R. Huxlin \\ Flaum Eye Institute, University of Rochester Medical Center, Rochester, New York 14642
}

The manipulation of visual perceptual learning is emerging as an important rehabilitation tool following visual system damage. Specificity of visual learning for training stimulus and task attributes has been used in prior work to infer a differential contribution of higher-level versus lower-level visual cortical areas to this process. The present study used a controlled experimental paradigm in felines to examine whether relearning of motion discrimination and the specificity of such relearning are differently influenced by damage at lower versus higher levels of the visual cortical hierarchy. Cats with damage to either early visual areas 17,18, and 19, or to higher-level, motion-processing lateral suprasylvian (LS) cortex were trained to perform visual tasks with controlled fixation. Animals with either type of lesion could relearn to discriminate the direction of motion of both drifting gratings and random dot stimuli in their impaired visual field. However, two factors emerged as critical for allowing transfer of learning to untrained motion stimuli: (1) an intact LS cortex and (2) more complex visual stimuli. Thus, while the hierarchical level of visual cortex damage did not seem to limit the ability to relearn motion discriminations, generalizability of relearning with a damaged visual system appeared to be influenced by both the areas damaged and the nature of the stimulus used during training.

\section{Introduction}

The intact, adult visual system is plastic and capable of learning from perceptual experience. The ability to generalize such learning across stimuli and contexts is important for adaptive function in our dynamic environment. Yet multiple studies have shown training-induced changes in visual function to be highly specific for trained stimulus attributes and retinotopic locations (Karni and Sagi, 1991; Sagi and Tanne, 1994; Ahissar and Hochstein, 1997). This suggested a critical role of lowlevel visual areas, with their precise retinotopic organization and specificity for basic stimulus attributes, in learning. However, subsequent work found that manipulations of task parameters, such as decreasing task difficulty (Ahissar and Hochstein, 1997), lowering discrimination precision (Jeter et al., 2009), and reducing the amount of training (Jeter et al., 2010), allowed learning to generalize across different stimulus modalities. Passive exposure and double training (Xiao et al., 2008) also resulted in transfer of learning across spatial locations. Thus, properties of the training stimulus and task can control generalizability and the neural substrates of learning (Sagi and Tanne, 1994).

\footnotetext{
Received Jan. 22, 2012; accepted Feb. 24, 2012.

Author contributions: K.R.H. designed research; M.D. and K.R.H. performed research; A.D. and K.R.H. analyzed data; A.D. and K.R.H. wrote the paper.

This work was supported by grants from the Schmitt Foundation and from the Research to Prevent Blindness Foundation. K.R.H is a recipient of the Research to Prevent Blindness Foundation's Lew R. Wasserman Merit Award. We thank Duje Tadin for his thoughtful, constructive comments on the manuscript, and Tracy Bubel for her excellent histology work.

The authors declare no competing financial interests.

Correspondence should be addressed to Krystel R. Huxlin, Flaum Eye Institute, University of Rochester Medical Center, 601 Elmwood Avenue, Box 314, Rochester, NY 14642. E-mail: huxlin@cvs.rochester.edu.

DOI:10.1523/JNEUROSCI.0316-12.2012

Copyright $\odot 2012$ the authors $\quad 0270-6474 / 12 / 325414-12 \$ 15.00 / 0$
}

Dosher and Lu suggested that the specificity of perceptual learning may arise from Hebbian reweighting of lower-level input channels by higher-level areas (Dosher and Lu, 1999, 2009). The reverse hierarchy theory (Hochstein and Ahissar, 2002; Ahissar and Hochstein, 2004) proposed learning as a top-down process modulated by task difficulty. Thus, easier tasks generalize more, as they primarily recruit higher-level stimulus representations. Meanwhile, with harder tasks, lower-level areas are recruited and their activity modified by higher-level areas, limiting transfer of learning. The "rule-based learning" model proposed that learning of task rules could instead occur at a centralized site, which then influences activity of multiple, lower-level inputs, allowing for transfer to occur (Zhang et al., 2010). Thus, if learning relies on an adaptive interplay between areas at different levels of the visual hierarchy (and possibly beyond), what happens when some of these areas are damaged? The present study begins to address this problem by asking: (1) Can specific visual perceptual abilities be relearned equivalently following damage to lower-level versus higher-level visual cortical areas? and (2) Are transfer properties of the relearned discriminations different for drifting gratings and random dot stimuli, and for animals with damaged early versus higher-level visual cortical areas? Here, we contrasted visual relearning in cats with permanent lesions of the lateral suprasylvian (LS) cortex or lesions of early visual areas 17, 18, and 19. LS cortex is a complex of higher-level visual areas (Palmer et al., 1978; Sherk, 1986a; Grant and Shipp, 1991; Sherk and Mulligan, 1993) functionally similar to medial temporal (MT) and medial superior temporal (MST) cortex in primates (Payne, 1993). Areas 17/ $18 / 19$ are considered feline homologues of primate V1/V2/V3 (Payne, 1993). Our results show a remarkable ability of the adult visual system with damage affecting early or higher-level 


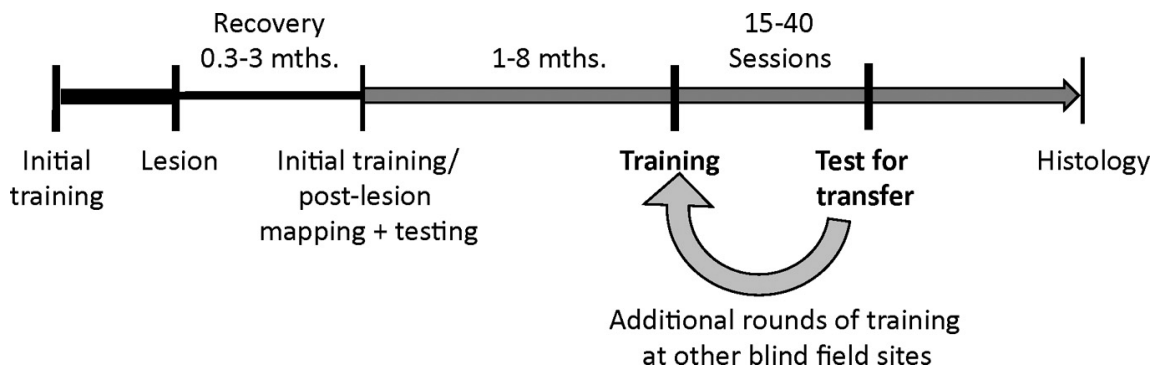

Figure 1. Experimental timeline. The thin black lines at the beginning of the trace indicate that eight cats were first trained to perform the required direction discrimination tasks (initial training) and then lesioned, while three cats received a cortical lesion first and then underwent initial behavioral training in intact regions of their visual field. The rest of the experimental timeline pertains to all 11 cats, which first underwent mapping of their visual deficit and pretraining tests. The cats were then trained at a first location in their impaired hemifields for 15-40 sessions at the rate of 1 session/d. This was followed by testing for transfer. Additional rounds of training at other locations within the impaired hemifield were performed in some of the animals, also followed by tests of transfer. Postmortem histology was performed at the end of experiment.

visual areas, to relearn discriminations it can no longer perform but was previously able to do. We also show an important role for both higher-level visual areas and the class of retraining stimulus in determining generalizability of motion discrimination relearning.

\section{Materials and Methods Subjects}

Eleven healthy, adult, male, domestic short-hair cats ( felis catus) were food restricted until their body weights ranged between 75 and $85 \%$ of normal. They then received the majority of their daily food in the form of pureed beef rewards for correct answers given during training and testing. Between training/testing sessions, the animals were supplemented with dry cat food, leaf lettuce, and vitamin pills to ensure stable body weight and good physical health. All experimental procedures were reviewed by the University of Rochester's Committee on Animal Research. They followed the recommendations of the Association for Research in Vision and Ophthalmology Statement for the Use of Animals in Ophthalmic and Vision Research, and the guide for the care and use of laboratory animals developed by the National Institutes of Health. Figure 1 provides a rough time line of the sequence of experimental procedures used in the present study. As a rule, animals underwent either initial behavioral training followed by a cortical lesion $(N=8)$ or a lesion first, followed by behavioral training $(N=3)$. Once animals had a lesion and had learned how to do the tasks at hand centrally and in their intact hemifields, they were treated identically, first undergoing behavioral mapping of motion discrimination across the visual field, followed by intensive discrimination training at a first location in the impaired hemifield. Once trained at this location, cats were tested there for transfer to untrained stimuli. Some then underwent a second or third round of training and testing for transfer at different, nonoverlapping locations in the impaired hemifield. Finally, postmortem histology was performed on the cats' brains to verify the location and extent of the cortical lesions.

\section{Initial behavioral training}

As mentioned earlier, eight cats underwent initial behavioral training before their lesion (six that then received LS lesions and two that received lesions of 17/18/19). This required that they undergo two separate surgeries, the first one to implant a head holder and subconjunctival eye coil (Pasternak and Horn, 1991; Huxlin and Pasternak, 2004). Once recovered from this first surgery, cats were trained to fixate and then to discriminate left-right drifting visual stimuli with controlled fixation. Once they had learned the task and exhibited stable thresholds for discriminating all three classes of stimuli used in the present study, these eight animals underwent a second surgery to make ibotenic acid lesions of visual cortex.

Three of the 11 cats underwent a single surgery to receive a head holder, a subconjunctival eye coil, and a unilateral cortical lesion (of LS cortex in two cats and areas $17 / 18 / 19$ in one cat). After recovering from the surgery, these three animals underwent initial fixation and discrimination training before going through with the mapping/training/testing phases of the experiment.

Initial fixation and discrimination training was identical for all cats, regardless of whether it occurred prelesion or postlesion. Cats were first taught to perform a left-right direction discrimination task using random dot stimuli and controlled fixation (see below) presented centrally until (1) they had learned the task and (2) their direction range (DR) thresholds reached stable levels ( $<10 \%$ coefficient of variation over a $5 \mathrm{~d}$ period). In cats, normal DR thresholds range between 11 and 35\% (Huxlin and Pasternak, 2004; Huxlin et al., 2008). Stimuli were then placed at 5-10 nonoverlapping, peripheral visual field locations of up to $20^{\circ}$ eccentricity (in both hemifields of vision for the eight prelesion cats and in the intact, ipsilesional hemifield in the three postlesion cats) to ensure that cats were able to perform the task with normal thresholds at a range of visual field locations. Generally, once cats had learned to perform the left-right direction discrimination task at one location, they transferred this learning effectively to other intact visual field locations. All training/testing sessions consisted of 200-300 trials and were always performed with controlled fixation. Animals typically performed one training or testing session per day, 5 d/week.

\section{Fixation control}

Eye movements of cats were monitored with subconjunctival eye coils, as described previously (Huxlin and Pasternak, 2004). During behavioral training and testing, the cats were placed inside a magnetic field generated by a set of $50 \mathrm{~cm}$ field coils and in front of a computer monitor displaying visual stimuli. Their heads were immobilized in front of the computer monitor using a cranially implanted head holder, and signal from their eye coil was detected using an eye coil phase detector (Riverbend Electronics). Eye position was calibrated before each daily testing session by rewarding the animal for keeping its gaze within an electronically defined square window centered $\pm 1.5^{\circ}$ on the fixation spot displayed on the computer monitor. The cats were trained to maintain fixation within this window for $1000 \mathrm{~ms}$. Ultimately, this allowed us to train them to discriminate peripherally presented visual stimuli, so that performance at different eccentric visual field locations could be mapped, before and after visual cortical lesions.

\section{Direction discrimination task}

Cats were taught to discriminate the left-right direction of motion of small, drifting visual stimuli displayed on a 19-inch ViewSonic monitor, located $42 \mathrm{~cm}$ in front of their eyes. The trial sequence (Fig. 2) started with the appearance of a small spot of light (the fixation spot) within a $\pm 1.5^{\circ}$ electronic window. Steady fixation of this spot for $1000 \mathrm{~ms}$ resulted in a tone and the appearance of the stimulus to be discriminated. Cats were required to maintain fixation on the fixation spot during stimulus presentation. After $500 \mathrm{~ms}$, both the stimulus and fixation spot disappeared. They were replaced by two "response spots" $\sim 10^{\circ}$ apart and located in the hemifield (upper or lower) opposite that which had contained the stimulus. Cats were taught to saccade to the rightmost response target for rightward moving stimuli, and to the leftmost response target for leftward moving stimuli. Each correct response was rewarded with a squirt of pureed beef. The sequence of presentation of rightwardmoving and leftward-moving stimuli was randomized. Incorrect responses resulted in a 3 s loud tone and no food reward. A break in fixation during stimulus presentation produced a brief, $1 \mathrm{~s}$ tone and the termination of the trial. To avoid positional biases, we used a correction procedure that detected sequences of three incorrect saccades to the same response target. The trial was then repeated until the animal made a correct response. Data obtained during correction procedures were re- 
corded but excluded from the final analysis used to compute a threshold and overall percentage correct performance for the session.

\section{Visual stimuli}

Gratings. Drifting, vertical, luminancemodulated sinewave gratings were used to measure the cats' contrast sensitivity for leftright direction discrimination. They were presented in a circular aperture $4^{\circ}$ in diameter and drifted either to the left or the right within this aperture. Grating spatial and temporal frequency were set to 0.3 cycles/degree and $6 \mathrm{~Hz}$ since these settings were previously found to elicit optimal contrast sensitivity in cats (Pasternak et al., 1995). The mean display luminance for this task was $20 \mathrm{~cd} / \mathrm{m}^{2}$ and stimulus duration exhibited a $250 \mathrm{~ms}$ raised cosine temporal envelope. Contrast thresholds were measured while cats discriminated the leftright direction of motion of the stimulus by varying luminance contrast of dark/light bars in the grating, computed as follows: grating contrast $=\left(L_{\max }-L_{\min } / L_{\min }+L_{\max }\right) \times 100$, where $L_{\min }$ and $L_{\max }$ refer to minimal and maximal luminance in the stimulus.

Random-dot stimuli. Random-dot stimuli, in which either the range of dot directions or the percentage coherence was varied, were used to measure the cats' DR and coherence thresholds, as described previously (Huxlin and Pasternak, 2004; Huxlin et al., 2008). Each stimulus consisted of small dots $\left(0.03^{\circ}\right.$ in diameter) presented within a circular aperture $4^{\circ}$ in diameter. To measure DR thresholds, the dots were repeatedly displaced in a range of directions chosen randomly from a uniform, limited distribution, centered on the rightward or leftward vector, with a new set of directions generated on each frame. To measure coherence thresholds, a different random dot stimulus was used in which a percentage of the dots moved coherently to the right or left, while the rest of the dots moved in a completely random set of directions. Individual dots always moved at a speed of $20^{\circ} / \mathrm{s}\left(\Delta t=13 \mathrm{~ms} ; \Delta x=0.26^{\circ}\right)$ for a limited lifetime of $250 \mathrm{~ms}$ (half the total stimulus duration). Dot luminance was set to $\sim 3.5$ $\log$ units above human detection threshold, on a relatively $\operatorname{dim}$ display $\left(0.1 \mathrm{~cd} / \mathrm{m}^{2}\right)$.

Threshold measurement. A staircase procedure was used to measure contrast, DR, and coherence thresholds. During each training/testing session, the stimulus set was varied along a selected dimension (luminance contrast for sinewave gratings, direction range, or percentage coherently moving dots for random dot stimuli) from the easiest to the most difficult using a staircase. Three consecutive correct responses caused an increase in stimulus difficulty (i.e., reduced contrast, increased range of dot directions, or decreased percentage of coherently moving dots), while a single incorrect response decreased it. The staircase used for DR threshold measurements consisted of $40^{\circ}$ steps between 0 and $355^{\circ}$. A threshold was calculated for each task, after each session, by fitting a Weibull function (Weibull, 1951) to the percentage correct performance at each stimulus level and computing the stimulus value (luminance contrast, direction range, or percentage coherence) corresponding to $75 \%$ correct performance. DR thresholds were expressed as a percentage of the maximal range of directions in which dots could move $\left(360^{\circ}\right)$ using the following formula: DR threshold $=\left(360^{\circ}-\mathrm{DR}\right.$ giving $75 \%$ correct performance $) /$ $360^{\circ} \times 100$. coherence thresholds.

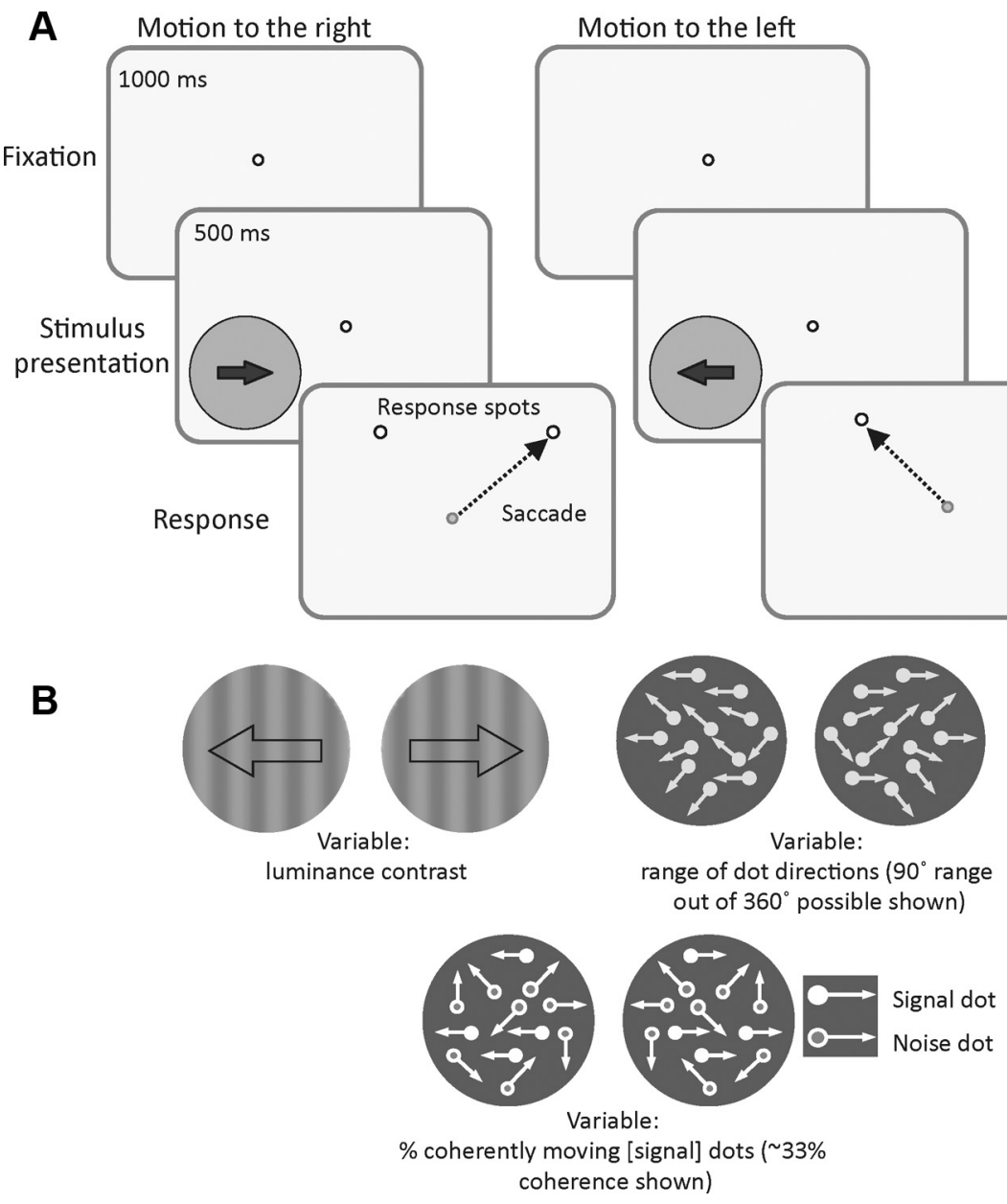

Figure 2. Behavioral paradigm. $\boldsymbol{A}$, During behavioral training and testing, cats were required to perform a left-right direction discrimination task that began when they precisely fixated a centrally placed target on a computer monitor in front of them for $000 \mathrm{~ms}$. A stimulus then appeared at a selected location in the central $40^{\circ}$ of their visual field, drifting either leftward or rightward s. Cats were required to maintain fixation on the central target during stimulus presentation. After $500 \mathrm{~ms}$, the stimulus monitor if the direction of motion of the stimulus had been to the right, and to the leftmost spot if the direction of motion

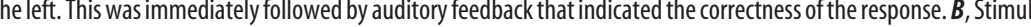
varying luminance contrast, we measured contrast thresholds for discriminating left-right motion direction. Light gray dots leftward or rightward vector were used to measure DR thresholds. Finally, light gray dots randomly distributed within a circular aperture over a black background and drifting either to the left or right (signal dots) or randomly (noise dots) were used to measure

\section{Cortical lesions}

Eight cats received unilateral LS lesions and three cats received unilateral lesions of contiguous, retinotopically corresponding regions of areas 17, 18, and 19. All surgeries were performed with cats placed in a Kopf stereotaxic apparatus under surgical anesthesia. For LS lesions, a rectangular craniotomy was made over one of the brain hemispheres (right in five cats and left in three cats). The craniotomy extended from $5 \mathrm{~mm}$ posterior to $15 \mathrm{~mm}$ anterior to the interaural line, and from 8 to $17 \mathrm{~mm}$ lateral to the midline. After removing the dura, 20-30 injections of 0.5-1 $\mu \mathrm{l}$ of sterile ibotenic acid (Sigma-Aldrich, $10 \mathrm{mg} / \mathrm{ml}$ in $0.1 \mathrm{M}$ PBS, pH 7.4) were made into both banks along the entire length of the LS sulcus to permanently destroy the gray matter of the posteromedial lateral suprasylvian, posterior lateral suprasylvian, anteromedial lateral suprasylvian, and anterolateral lateral suprasylvian areas. All injections were made using a 10 or $25 \mu \mathrm{l}$ Hamilton syringe fitted with a 32 gauge, beveled needle. Injection sites were spaced at intervals of $1.7 \mathrm{~mm}$ across the cortical surface, starting $3 \mathrm{~mm}$ posterior to the interaural line and ending $\sim 14 \mathrm{~mm}$ anterior to the interaural line. 
For lesions of areas 17/18/19, a rectangular craniotomy was made over the left hemisphere in two cats and the right in one cat, extending from 7 $\mathrm{mm}$ posterior to $10 \mathrm{~mm}$ anterior to the interaural line, and from 0 to 10 $\mathrm{mm}$ lateral to the midline. After removing the dura, $\sim 30$ injections of

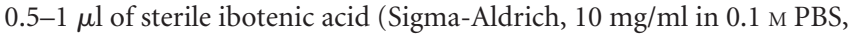
$\mathrm{pH}$ 7.4) were made into the gray matter of the medial and lateral aspects of the posterior lateral gyrus (targeting areas 17 and 18) and the medial aspect of the posterior suprasylvian gyrus (targeting area 19). All injections were made using a 10 or $25 \mu \mathrm{l}$ Hamilton syringe fitted with a 32 gauge, beveled needle. Injection sites were spaced at intervals of $1.7 \mathrm{~mm}$ across the cortical surface. In cats $4-008$ and $8-002$, the injections were started $5 \mathrm{~mm}$ posterior to the interaural line and ended $\sim 7 \mathrm{~mm}$ anterior to the interaural line, affecting the representation of both upper and lower quadrants of the contralateral visual hemifield (Tusa et al., 1978, $1979,1981)$. In cat 6-010, bone structure and brain morphology forced us to start injections at $\sim 3 \mathrm{~mm}$ posterior to the interaural line. As a result, his lesion (see Fig. $3 A$ ) was smaller than that in the other two cats in this group, primarily affecting the lower quadrant representation of the contralateral visual hemifield (a prediction verified by mapping of his visual field defect; see Fig. 4).

The separation of the injections and the amount and concentration of ibotenic acid injected were previously shown to produce large, contiguous lesions of the desired visual cortical areas (Huxlin and Pasternak, 2001, 2004; Huxlin et al., 2008). The animals' EKG, $\mathrm{CO}_{2}$, and temperature were constantly monitored during the surgery and kept at physiological levels. After completing the injections, the dura and bone flap were pulled back into place. The head holder was reconstructed and the muscles, subcutaneous tissues, and skin were sutured around it before the animals were recovered under veterinary supervision.

\section{Postlesion behavioral testing and training}

\section{Assessment of visual deficit}

During the first 3-4 d after their lesion surgery, all LS-lesioned cats exhibited a stereotyped, rotational behavior toward the side of the lesion, which disappeared by the fifth day postlesion. No such behavior was observed in cats with area 17/18/19 lesions. Behavioral testing began a few weeks to 3 months postoperatively and was dictated by the postsurgical health of each animal. DR thresholds were first measured centrally and at several locations in the visual hemifields ipsilateral to the lesion (i.e., in the intact hemifields) with a $4^{\circ}$ stimulus and controlled fixation. For cats that had been trained prelesion, this allowed us to verify that DR thresholds in intact portions of the visual field remained at preoperative levels (i.e., the cat had not forgotten how to fixate or how to do the task, and had not sustained unplanned damage to both cortical hemispheres). DR thresholds were then measured in the hemifields contralateral to the lesioned hemisphere, out to an eccentricity of $\sim 20^{\circ}$ (see Fig. 4 ). To minimize learning effects during this mapping procedure, no more than two 200-trial sessions were run at each visual field location.

\section{Selecting impaired visual field locations for postlesion training}

For each cat, one or more locations in the hemifield contralateral to the lesion were selected for visual retraining. Criteria for choosing these sites were that the cat should be unable to discriminate the global direction of motion of coherently moving dots at these locations postlesion and that DR thresholds should also be abnormal at nonoverlapping sites surrounding these locations. Each of the T's in Figure 4 indicate locations for training in each cat.

In addition to measuring DR thresholds at the chosen training locations, we also measured one or both of the other two outcome measures in this study: coherence thresholds and luminance contrast thresholds for left-right direction discrimination. This allowed us to assess the effects of loss of LS cortex or areas 17/18/19 on the gross direction discrimination of drifting gratings and random dot stimuli. Visual measurements performed in the impaired hemifield of each cat were repeated at corresponding "control" locations in the ipsilesional (intact) hemifield, sometimes before the onset of visual retraining and sometimes after retraining (see Figs. 5-8, white histogram bars). This provided an internal control for the animals' motivational and perceptual states, and enabled us to estimate normal threshold performance for each cat at corresponding visual eccentricities.

\section{Postlesion retraining in impaired hemifields: procedures and tests for transfer of learning}

After mapping the visual deficit using DR thresholds, one or more locations in the impaired hemifield of each cat underwent direction discrimination training using either (1) random dot stimuli in which the range of dot directions was varied between 0 and $355^{\circ}$ in a staircase procedure (as described above), or (2) drifting, vertical sinewave gratings in which luminance contrast was varied in a staircase procedure (described above). A total of 13 nonoverlapping, impaired visual field locations were trained among the eight LS-lesioned cats, while seven different locations were trained among the three cats with 17/18/19 lesions. In LS-lesioned cats, eight of the selected 13 blind field locations were first trained on gratings, while the remaining five underwent their first round of training on dots. In cats with 17/18/19 lesions, three of the seven selected locations were first trained using gratings, while the rest were first trained on dots. Cats performed 200-300 trials of the left-right direction discrimination task per day at a single location until their threshold performance stabilized within the normal range, relative to performance at corresponding locations in the intact hemifield. Thresholds were defined as stable when their coefficient of variation was $<10 \%$ of the mean over the last five data points. Between 15 and 40 training sessions were required for recovery to stable, normal levels of performance and there appeared to be no systematic correlation between this number and stimulus or lesion type.

Once DR or contrast thresholds had recovered to normal levels at the retrained locations, we assessed whether this recovery had transferred to the discrimination of untrained stimuli. Cats trained using random dot stimuli underwent measurement of contrast sensitivity and coherence thresholds. Cats retrained using drifting sinewave gratings were tested using random dot stimuli to assess whether their DR and coherence thresholds had decreased at the same visual field locations, suggesting an improvement in the ability to discriminate these untrained stimuli.

Whenever a deficit was observed for discrimination of the transfer stimulus, that discrimination was then trained to ensure that it could recover. Retraining with the transfer stimulus category was continued until thresholds reached control levels, determined from the animals' performance at corresponding locations in their intact hemifield of vision.

\section{Retinotopic specificity of global motion discrimination training}

To assess whether learning to discriminate random dot stimuli with a large range of dot directions was limited to the trained locations, we measured DR thresholds at several, nonoverlapping sites within the impaired or intact hemifields, at different distances away from the trained locations. For postlesion cats, the DR threshold measured at each impaired hemifield location before the onset of retraining (during the postlesion mapping sessions) was then subtracted from the post-training DR threshold at the same site to generate an estimate of the training effect (see Fig. 9C,D, DR change from pretraining levels). Only locations significantly affected by the lesion (with starting DR thresholds $>50 \%$, which was more than two SDs away from the mean in the same cats' intact hemifields of vision) were chosen for this retinotopic specificity analysis.

\section{Postmortem histology and lesion reconstruction}

At the end of the experiments, cats were sedated with an intramuscular injection of $20 \mathrm{mg} / \mathrm{kg}$ ketamine before undergoing euthanasia with an overdose of sodium pentobarbital (100 mg/kg, intraperitoneally). Once breathing had stopped and reflexes had disappeared, the cats were perfused through the heart with $3 \%$ paraformaldehyde and $0.1 \%$ glutaraldehyde in $0.1 \mathrm{~m}$ phosphate buffer (PB), $\mathrm{pH}$ 7.4. After dissection, the brain was placed into $30 \%$ sucrose in $0.1 \mathrm{M} \mathrm{PB}$ until adequately cryoprotected, at which stage, serial, frozen sections were cut at a thickness of $40 \mu \mathrm{m}$. Alternate sections were reacted for cytochrome oxidase (CO), a marker of neuronal activity that can be used to precisely delineate brain regions where neurons have been killed, and for Nissl substance with cresyl vio- 

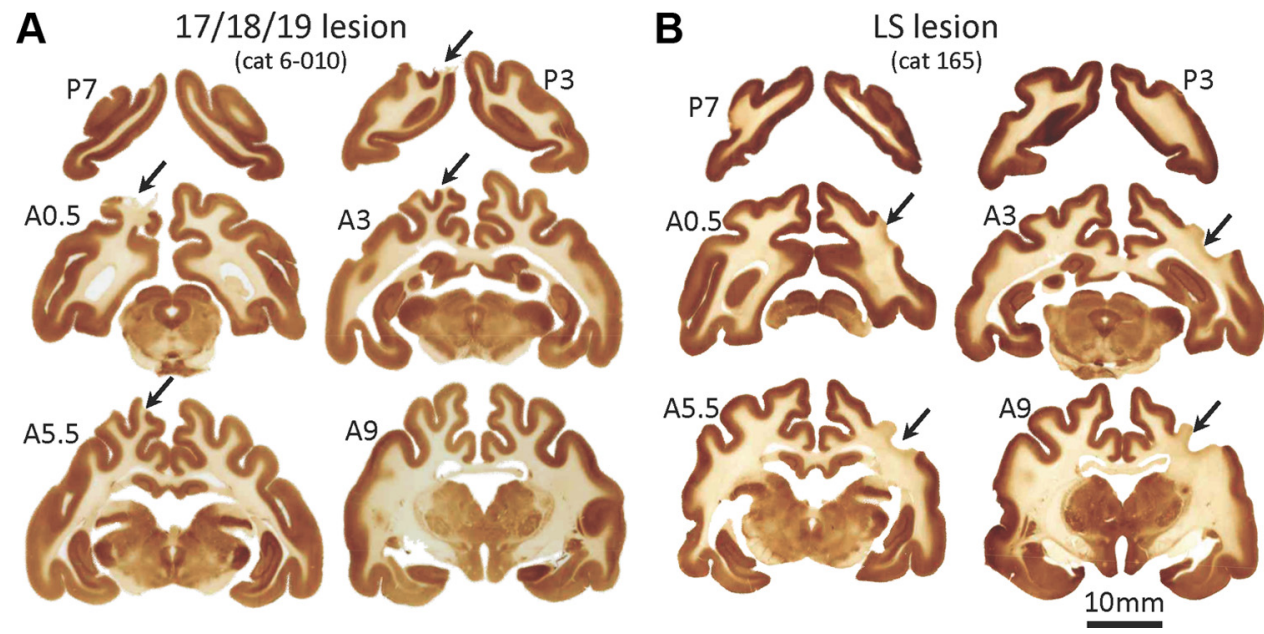

Figure 3. Anatomy of brain lesions. $A$, Coronal brain sections from one cat with a lesion of areas $17 / 18 / 19$ (cat $6-010$ ) in the left hemisphere. Sections shown were sampled at regular intervals between a region $7 \mathrm{~mm}$ posterior (P7) to $9 \mathrm{~mm}$ anterior (A9) to the interaural line. They were stained for cytochrome oxidase reactivity to show areas of intact gray matter (brown) and, by inference, the location and extent of the lesion (arrows). The lesion can be recognized in comparison with the intact hemisphere (right in this cat), as a region of missing gray matter. $\boldsymbol{B}$, Coronal brain sections from one of the cats with a lesion of $L S$ cortex, showing a large region of missing gray matter in the right hemisphere. Note, however, that the areas of early visual cortex damaged in cat $6-010$ ( $A$ ) are intact in cat 165 , and vice versa.

let. For the $\mathrm{CO}$ reaction, free-floating brain sections were incubated at $37^{\circ} \mathrm{C}$ for $3-4 \mathrm{~h}$ in a solution of $0.06 \%$ diaminobenzidine tetrahydrochloride (Sigma-Aldrich), $0.03 \%$ cytochrome C (type III, Sigma-Aldrich) and $4.4 \%$ sucrose in $0.1 \mathrm{M} \mathrm{PB}$. Once the sections reached the appropriate dark-brown coloring, they were rinsed and mounted onto subbed slides for analysis. The Nissl stain was used to verify the extent of the lesions, identify cortical layers, and help distinguish borders between some cortical areas.

The cortical areas damaged by the ibotenic acid injections were identified from CO-stained sections in which damaged cortex characteristically lacks brown staining in the gray matter (Huxlin and Pasternak, 2001). The damaged cortical areas in CO-stained sections were then identified by comparison with the visual cortical maps published by Tusa and colleagues (Tusa et al., 1978, 1979, 1981; Tusa and Palmer, 1980) and Palmer and colleagues (Palmer et al., 1978).

\section{Statistical methods}

For DR, coherence and contrast thresholds, the mean and the SD of the parameters of interest were compared between locations in the intact and impaired hemifields, and as a function of training, with Student's $t$ tests. All inferential tests were two-tailed and a $p<0.05$ was considered statistically significant.

\section{Results}

\section{Effect of damaging LS cortex or areas $17 / 18 / 19$ on motion} perception

All cats that received ibotenic acid lesions exhibited a region of decreased CO activity unilaterally, either on the banks of the lateral suprasylvian sulcus (LS cortex) or in the targeted regions of areas $17 / 18 / 19$ (Fig. 3). According to the maps of Tusa and colleagues (Tusa et al., 1978, 1979, 1981), an almost identical representation of the contralateral visual field was damaged by our lesions in areas 17, 18, and 19 of cats $6-010,4-008$, and $8-002$. Both lesion types caused deficits in left-right DR integration thresholds (Figs. 4, 5C, 6D-F). Thus we used DR thresholds to map the extent and severity of the visual deficit in each animal out to $\sim 20^{\circ}$ eccentricity (Fig. 4 ). Though all cats exhibited deficits in DR thresholds in the hemifield contralateral to the lesion, the precise extent and severity of the deficit differed among animals.
Among animals with lesions of low-level visual cortex in areas $17 / 18 / 19$, cats $4-008$ and $8-002$ exhibited the largest, contiguous area of deficit. Lesion of areas $17 / 18 / 19$ was smallest in cat 6-010 (Fig. 3A), likely explaining the smaller visual deficit in this cat (Fig. 4). Among animals with lesions of LS cortex, cats 2-004, 6-004, 008, 4-017, and 165 (whose COstained brain sections are shown in Fig. $3 B$ ) exhibited the largest contiguous deficits. Nevertheless, the contralesional hemifields of all cats contained locations where the deficit as defined by DR thresholds was maximal (Fig. 4, dark gray circles). Some areas of sparing were seen in all cats, especially within the central $5^{\circ}$ of vision. This was fortuitous for placement of the response spots in those locations.

In addition to impaired DR thresholds, all three cats with $17 / 18 / 19$ lesions demonstrated high contrast thresholds (Figs. 5B, $6 B$, dark histogram bars) and high coherence thresholds (Fig. $6 H$ ). In contrast, as shown previously (Pasternak et al., 1989; Rudolph and Pasternak, 1996), cats with unilateral lesions of LS cortex had normal contrast thresholds (Fig. 6C), but abnormal DR (Figs. 5C, 6F) and coherence thresholds (Fig. 6I) for random dot stimuli moving at the same speed and at the same visual field locations.

\section{Effect of retraining on discrimination of trained stimuli}

In the context of damage to higher versus lower levels of the visual cortical hierarchy, a question arose as to whether it is possible to retrain the visual system to recover the associated deficits in either case. As shown in Figure 6 B, damage to areas 17/18/19 caused an average 28 -fold increase in contrast thresholds for discriminating the direction of motion of drifting sinewave gratings. When these cats underwent repetitive contrast sensitivity training in their impaired hemifield, they demonstrated a gradual improvement in thresholds within 15-30 training sessions or 3000-6000 trials (see Fig. $5 B$ for an example of learning on this task by cat $6-010$ ). By the end of training, contrast thresholds reached a level that was not significantly different from contrast thresholds at equivalent locations in the intact hemifield of the same animals (Fig. $6 \mathrm{~B}$ ). Consistent with previous reports (Pasternak et al., 1989; Rudolph and Pasternak, 1996; Huxlin and Pasternak, 2004), we found that 
A
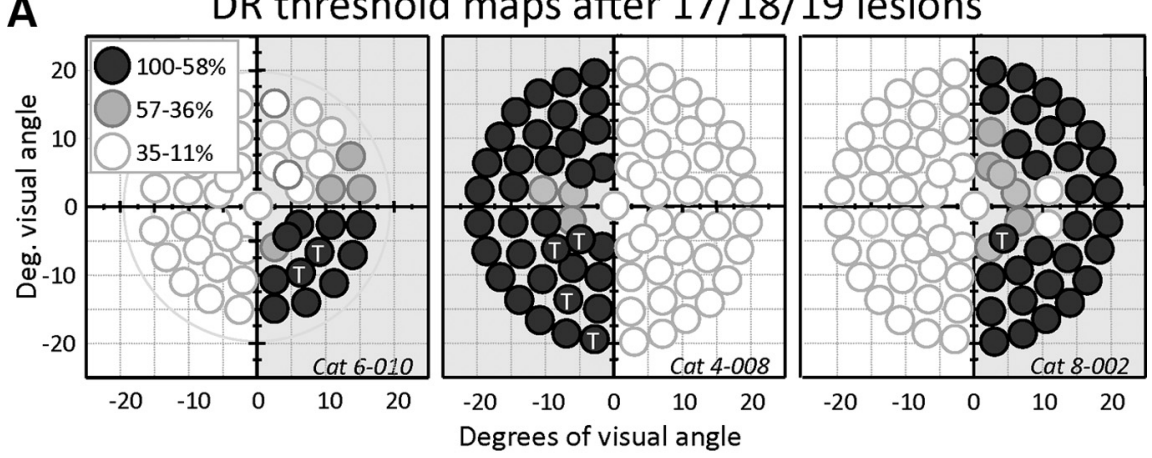

B
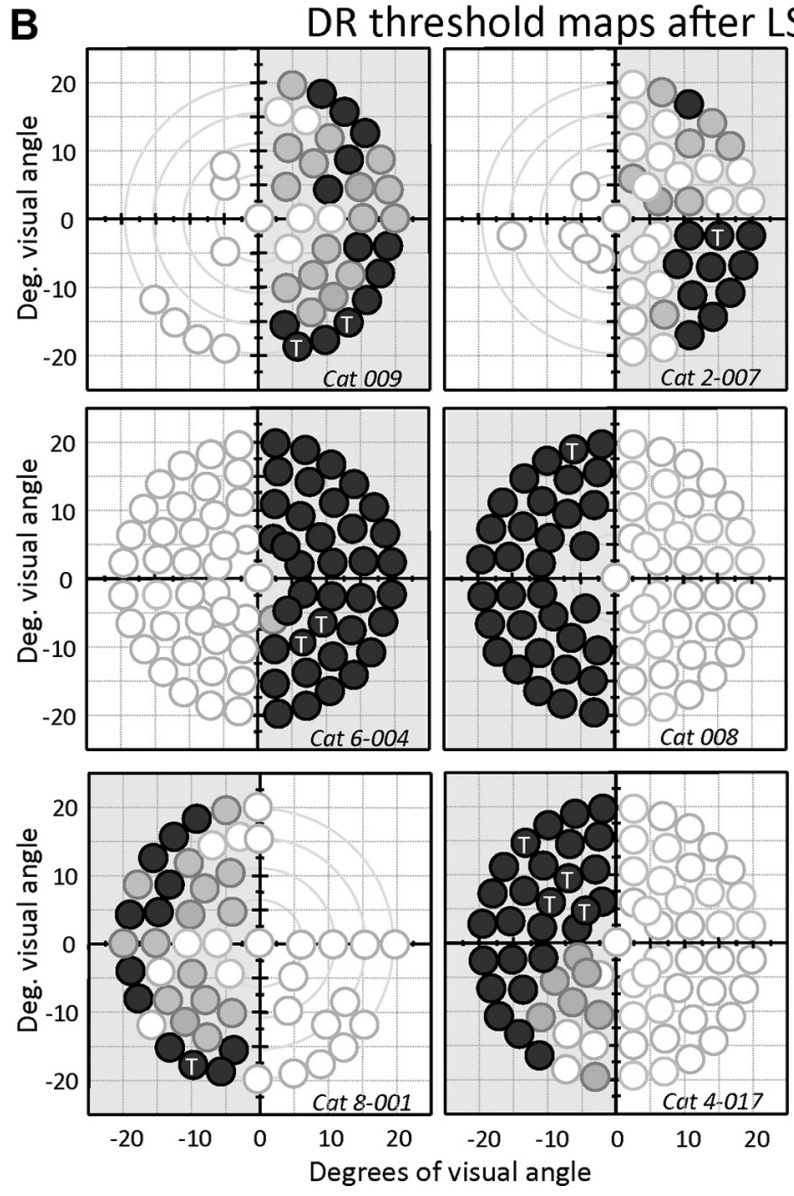

Figure 4. Effect of cortical lesions on DR thresholds: a mapping study. Maps of visual field space illustrating the location, size and shape of visual stimuli used to measure DR threshold performance at different, nonoverlapping, visual field locations in each cat. Animals are separated according to lesion type ( $\boldsymbol{A}$, cats with $17 / 18 / 19$ lesions and $\boldsymbol{B}$, cats with LS lesions). Axes are labeled in degrees of visual angle. As defined previously, normal DR thresholds in the cat range from 11 to $35 \%$ (white circles). DR thresholds $>35 \%$ were only seen contralateral to the brain lesion (right or left impaired hemifields denoted by light gray shading) in both groups. Deficits varied from mild (DR thresholds between 36 and $57 \%$, gray circles) to severe (DR thresholds between 58 and $100 \%$, black circles). The area of visual field covered by the most severe deficits differed among animals, and was largely consistent with the physical size of the brain lesion. For instance, cat 6-010 had one of the smallest areas of brain damage in corresponding regions of areas 17, 18, and 19 (Fig. $3 A$ ) and exhibited only a quadrant deficit. In contrast, cat 165 (Fig. 3B), who exhibited abnormal DR thresholds over practically his entire left hemifield of vision, had an almost complete destruction of LS cortex in his right hemisphere (Fig. 3B).

LS lesions did not affect contrast sensitivity for direction discrimination of low-spatial-frequency sinewave gratings drifting at $20 \%$ s (Fig. 6C). Consequently training these cats on drifting gratings did not result in any significant training-induced improvements in contrast thresholds (Fig. 6C).

All cats in the present study exhibited poor DR (Fig. 6E,F) and coherence thresholds (Fig. 6H,I). However, when trained repetitively to discriminate the global direction of motion of DR stimuli in a previously untrained location, cats with $17 / 18 / 19$ and LS lesions progressively improved their DR thresholds (Fig. 6E,F). The DR relearning in cats with LS lesions replicated previous work from our laboratory (Huxlin and Pasternak, 2004; Huxlin et al., 2008). Similarly, when training was administered using stimuli in which coherence was varied, coherence thresholds progressively improved back to normal levels (Fig. $6 \mathrm{H}, \mathrm{I}$ ). An important aside here is that lesioned animals could perform all three tasks centrally and in the intact hemifield of vision following their lesion. From the cats trained before their lesion, it was possible to assess that thresholds were not significantly different in the postlesion, intact hemifields of vision (which always included the foveal representation) compared with prelesion values measured both centrally and peripherally (see Fig. $9 A, B$, DR thresholds; results not shown for coherence and contrast sensitivity thresholds).

With respect to visual discriminations in the contralesional visual hemifield, we should also point out that there was a critical requirement for training to elicit recovery postlesion. This was demonstrated previously following LS lesions (Huxlin and Pasternak, 2004) and presently for the first time for lesions of areas 17/18/19. Neither the passage of time nor passive exposure to visual stimulation recovered motion discrimination at contralesional sites distant from the retrained locations in any of our animals. Thus, just as in humans with primary visual cortex damage (Zhang et al., 2006a,b), spontaneous improvements in vision (or at least visual motion perception) do not occur in cats with early visual cortex lesions.

Therefore, regardless of whether they had sustained a lesion of lower-level visual areas (17/18/19) or of higher-level visual areas (LS cortex), all cats were able to regain normal sensitivity (thresholds) for discriminating the left-right direction of motion of the stimuli used for training. More precisely, specific retraining with luminance-modulated sinewave gratings recovered contrast sensitivity for direction. Specific training with random dot stimuli comprising multiple directional vectors centered on a mean direction recovered motion integration thresholds. Training with random dot stimuli in which only some dots moved coherently to the right or left recovered coherence thresholds back to normal levels of performance, as measured in the intact hemifield postlesion. 
Generalization of visual motion relearning following damage

Effect of contrast sensitivity training on $D R$ and coherence thresholds

Once we established that all motion discriminations of interest could be recovered to normal threshold levels of performance following 17/18/19 lesions and LS lesions in adult cats, we then asked whether practice on a contrast sensitivity task could improve motion integration (i.e., DR thresholds or coherence thresholds) (Fig. 7A). Baseline thresholds were measured on 2 consecutive days in seven of the 11 cats (four with LS lesions and three with 17/18/19 lesions). These cats were then trained using sinewave gratings for $40 \mathrm{~d}$ at an impaired hemifield location where DR thresholds were $>90 \%$. Cats with LS lesions did not exhibit a contrast sensitivity deficit and, likely because they had been fully trained on this task before the lesion, they did not show further improvements in contrast thresholds with practice (Fig. $6 \mathrm{C}$ ). The three cats with lesions of areas 17/18/19 exhibited decreased contrast sensitivity for direction (Fig. 6B), and were first retrained on the contrast sensitivity task until they regained normal contrast thresholds. Across the seven cats trained for this experiment, contrast thresholds averaged $2.2 \pm 0.57 \%$ at the end of training, which was not significantly different ( $p=0.347$, Student's $t$ test) from contrast thresholds measured at corresponding locations in the same cats' intact hemifields $(1.7 \pm 0.86 \%)$. Nor were there significant differences between cats with different lesions.

At this point, DR and coherence thresholds were remeasured at the trained locations to assess whether intensive practice on the contrast sensitivity task affected motion integration or coherence thresholds. Neither threshold improved from levels measured initially following either type of lesion (Fig. 7). DR thresholds averaged $96 \pm$ $6 \%$ in $17 / 18 / 19$-lesioned cats (Fig. $7 B$ ) and $95 \pm 9 \%$ in LSlesioned cats (Fig. $7 C$ ) over the first $3 \mathrm{~d}$ of testing after the end of practice with gratings. Similarly, coherence thresholds averaged $72 \pm 18 \%$ in $17 / 18 / 19$-lesioned cats (Fig. $7 D$ ) and $72 \pm 28 \%$ in LS-lesioned cats (Fig. 7E). These values were not significantly different ( $p$ values ranging from 0.353 to 0.811 , Student's $t$ test) from the thresholds obtained over the first $2 \mathrm{~d}$ of testing after the lesion (before training with gratings). Thus, although the task was the same, the visual field locations stimulated and attended to were the same, and dots moved coherently during most of the testing, grating-trained cats were unable to discriminate the global direction of motion in random dot stimuli. However, when these same cats were subsequently trained using random dot stimuli either with a variable range of dot directions or variable coherence levels, their DR and coherence thresholds improved. After $\sim 20 \mathrm{~d}$ of training, they reached normal levels of performance $(\mathrm{DR}$ thresholds $=30 \pm 6 \%$; coherence thresh- olds $=31 \pm 4 \%)$. Though initial training with grating stimuli did not improve DR or coherence thresholds at the trained locations, it did not impair nor benefit the animals' ability to eventually relearn to discriminate the direction of motion of random dot stimuli.

\section{Effect of DR training on contrast sensitivity}

Our next question was whether relearning to extract global direction of motion from a patch of random dots would generalize to simpler motion discriminations comprising a single directional vector. Specifically, we asked whether global motion discrimination training in the impaired hemifield improved contrast sensitivity for discriminating the direction of motion of a moving sinewave grating. Because contrast sensitivity for our left-right direction discrimination task was only affected in cats with lesions of areas 17/18/19 (Fig. $6 B, C$ ), a new location was selected in these animals where DR thresholds were $>90 \%$. Cats underwent training using random dots in which the range of dot directions was varied and, once their DR thresholds reached normal levels, we retested contrast sensitivity at the same location (Fig. 8A). Contrast thresholds improved dramatically as a function of DR training, averaging $1.8 \pm 0.9 \%$, which is not significantly differ- 
A

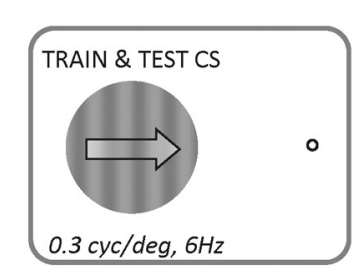

D

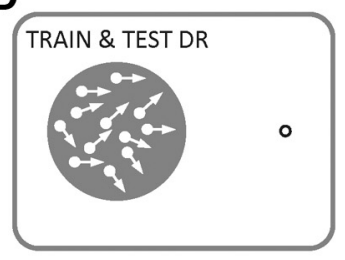

G

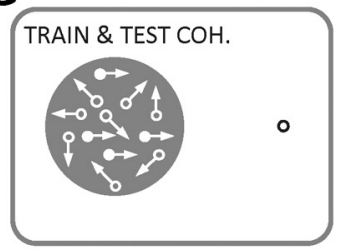

B
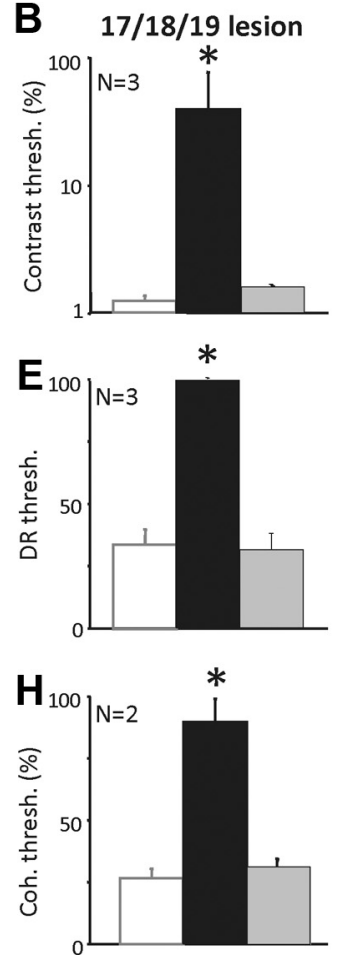

C

LS lesion
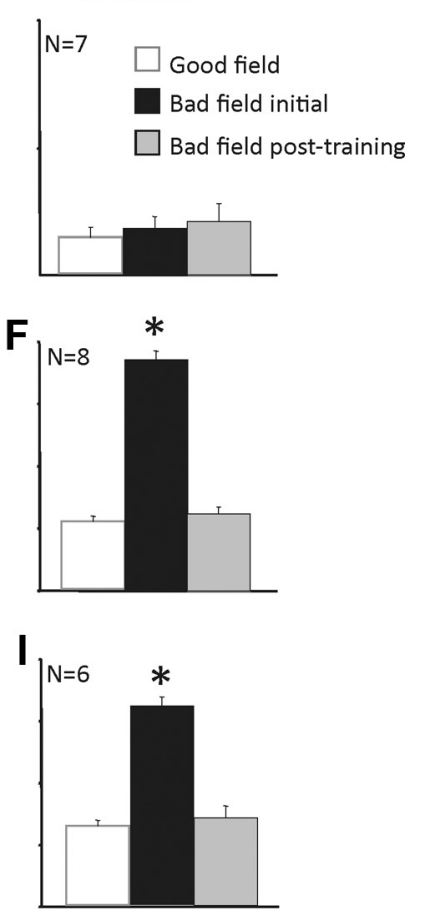

Figure 6. Training improves discrimination thresholds for trained stimuli, regardless of lesion type.A, In this first experiment, cats were trained to discriminate the left-right direction of motion of sinewave gratings at 0.3 cycles/degree spatial frequency and drifting at $6 \mathrm{~Hz}$. A staircase was used to progressively decrease stimulus contrast in each training session. B, Plot of contrast threshold in cats with 17/18/19 lesions in the intacthemifield (good field), in the impaired hemifield beforetraining (bad field initial), and at the samelocation post-training (bad field post-training). Training gradually improved contrast thresholds at the trained locations in the contralesional visual hemifield (Fig. 5) until contrast thresholds were no longer significantly different from those in the intact hemifield of vision. C, Cats with LS lesions did not exhibit a significant deficit in contrast sensitivity for direction (compare black and white bars) and additional practice of this task in the impaired hemifield did not further improve the animals' contrast thresholds (gray bar). $\boldsymbol{D}$, At a different, nonoverlapping location in their impaired hemifield, the same cats were trained to discriminate the left-right direction of motion of random dot stimuli in which the range of dot directions was varied from easier to harder levels in each session. E, Before training, cats with 17/18/19 lesions exhibited significantly raised DR thresholds (black bars). Training improved these abnormal thresholds back to normal levels (gray bars).F, A very similar pattern of postlesion deficit and training-induced recovery of DR thresholds was seen in cats with $L S$ lesions. G, Finally, at yet another location in the impaired hemifield of vision, cats were trained to discriminate the left-right direction of motion of random dot stimuli in which the proportion of coherently moving dots was varied using a staircase procedure in each session. $\boldsymbol{H}$, Beforethe onset of training, cats with lesions of areas 17/18/19 exhibited significantly higher coherence thresholds in the contralesional (black bars) versus the ipsilesional hemifield (white bars). Just as in $\boldsymbol{B}$ and $\boldsymbol{E}$, specific training gradually improved impaired coherence thresholds back to normal levels (gray bars) at the trained locations. I, A similar pattern of postlesion deficit and training-induced recovery of coherence thresholds was seen in cats with $L S$ lesions. The number of cats used in each portion of the experiment is shown as $N$. All values given are means \pm SEM. ${ }^{*} p<0.05$, paired Student's $t$ tests relative to intact hemifield values. CS, Contrast sensitivity; Coh, coherence.

ent from contrast thresholds at equivalent locations in the intact hemifields of the same cats (Fig. $8 B$ ).

\section{Effect of lesion type on transfer of DR training to coherence thresholds}

Our third manipulation was designed to assess whether DR training improved not only contrast sensitivity, but also the extraction of coherent motion signals from noise. Cats with lesions of either areas $17 / 18 / 19(N=3)$ or LS cortex $(N=5)$ were retrained to discriminate the global direction of motion of random dot stimuli. Once they had recovered normal DR thresholds, their coherence thresholds were remeasured (Fig. 8C,D). The three cats with lesions of areas 17/18/19 showed effective transfer of learning, with coherence thresholds averaging $82.4 \pm 17.4 \%$ pre-DR training (highly abnormal), decreasing significantly to $30.8 \pm 7 \%$ after DR training (Fig. $8 C$ ). This was not significantly different from normal threshold performance in the intact hemifield (26.5 $\pm 7 \%$ ), suggesting that coherence thresholds had recovered to almost normal levels after DR training.

In contrast, when five of the cats with LS lesions underwent DR retraining, they recovered normal DR thresholds, but their coherence thresholds remained abnormally high, averaging $73.3 \pm 20.1 \%$ (Fig. 8D). This was not significantly different from the animals' coherence thresholds at these locations before DR training (Fig. 8D, black bar; $75.6 \pm$ $10.1 \%)$. It was also significantly higher than coherence thresholds at corresponding locations in the intact hemifield (Fig. $8 D$, white bar; $30.2 \pm 6.5 \%$ ). The present result is consistent with that reported in our previous publication on three LS-lesioned cats (Huxlin and Pasternak, 2004). Subsequent training of the five LS-lesioned cats with random dot stimuli in which coherence was varied in a staircase procedure improved their thresholds to $33.5 \pm 11.1 \%$, on average not significantly different from that for the intact hemifield. Thus, coherence thresholds were not permanently affected by damage to LS cortex. Overall, comparison of Figure $8 C$ and $8 D$ shows a clear effect of lesion site (17/18/19 vs LS cortex) on the ability to transfer learning of a motion integration task to one requiring efficient extraction of coherent motion signals from noise.

\section{Effect of lesion type on retinotopic specificity of global motion learning} After brain damage, the ability of DR learning to generalize across visual field locations, which was evident prelesion (Fig. 9A), remained in the intact hemifield (Fig. 9B), but decreased significantly in the blind hemifield (Fig. 9C,D). Cats with both lesion types exhibited maximal improvement at trained locations in their impaired hemifields (Fig. 9C). Here, improvement was defined as the increase in DR threshold relative to the threshold measured at the same location postlesion, but before the onset of training. Only locations where DR thresholds were $\geq 50 \%$ were used in this analysis. DR thresholds $\geq 50 \%$ were selected for this analysis because they were $>2$ SDs away from normal thresholds prelesion and in the intact hemifield of vision postlesion. As shown in Figure 9C, the amount of training-induced improvement decreased with increasing distance away from the trained, blind field locations, and it did so faster for cats with lesions of areas 17/18/19 than in cats with LS lesions. Also notable was the fact that eccentricity (relative to fixation) seemed to play a less significant role in decreasing performance than distance away from the trained location (much greater slopes in Fig. 9C than 9D).

An important question here is whether the retinotopic specificity of relearning observed in impaired hemifields was due to these locations receiving more training postlesion than locations subserved by intact cortical circuitry. Seven of the animals used 
for the retinotopy data in Figure 9 underwent intensive training to perform the tasks at hand prelesion. The remaining animals learned the tasks postlesion centrally and in their intact visual hemifield. Both groups required $\sim 30-40$ training sessions before DR thresholds stabilized at their initial, intact training location. Once learning occurred in the intact hemifield, all animals were able to generalize this learning to other, intact visual field locations (Fig. 9A, $B$ ), regardless of when this initial training happened (prelesion or postlesion) and regardless of the type of lesion they sustained. Yet, when a location in the impaired hemifield was retrained postlesion, relearning DR thresholds took approximately the same number of training sessions as initial learning, but it became more retinotopically specific within the impaired hemifield (Fig. 9C,D). Thus, location specificity of visual relearning appears to be a property of impaired hemifields and visual cortex damage, rather than a consequence of increased amounts of training in impaired hemifields postlesion.

\section{Discussion}

Specificity to stimulus and visual field location is often used to infer the relative contribution of lower-level versus higherlevel areas to perceptual learning. Here, we asked whether a visual system with damage to either lower or higher levels of the cortical hierarchy could be retrained to perform a simple, left-right direction discrimination task, and we assessed specificity of relearning for stimuli and locations trained. We find that visual cortex damage does not prevent relearning of motion discriminations. However, generalizability of relearning was influenced by both the hierarchical locus of damage and the type of stimulus used.

Consistent with previous studies that damaged areas 17 or 18 individually (Pasternak and Maunsell, 1992; Pasternak et al., 1995), combined damage to areas 17 , 18 , and 19 impaired performance on all visual motion discriminations tested here. This result confirms suggestions from anatomy (Symonds and Rosenquist, 1984; Sherk, 1986a,b; Huxlin and Pasternak, 2001) and electrophysiology (Dreher et al., 1996; Lee et al., 1998), that areas 17, 18 , and 19 together provide the majority of basic signals needed by LS cortex to correctly extract global motion information. However, here we show that cats with lesions of early visual cortex, just like humans with V1 damage (Huxlin et al., 2009), can be retrained to discriminate global motion direction, and their con-

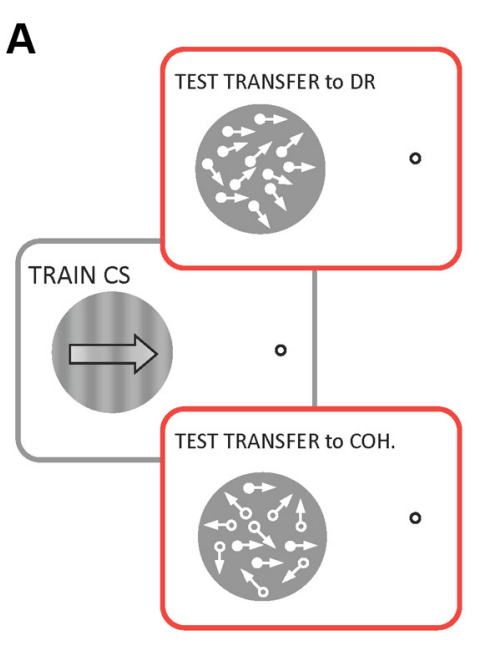

B

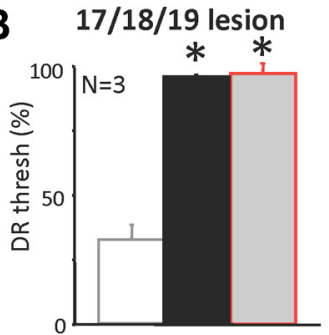

C

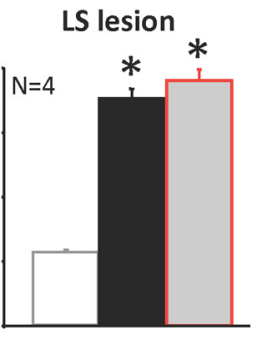

D

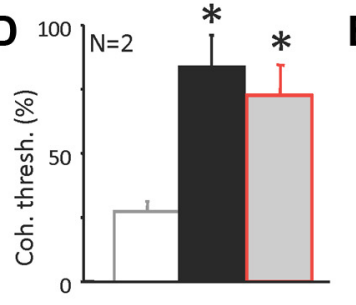

$\mathbf{E}_{\mid \mathrm{N}=3}$

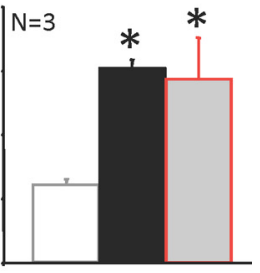

$\square$ Good field
$\square$ Bad field initial
$\square$ Bad field + CS training

Figure 7. Training to discriminate direction of sinewave gratings does not improve global motion thresholds. $\boldsymbol{A}$, In this experiment, postlesion cats were retrained to discriminate the left-right direction of motion of drifting sinewave gratings at one location in their impaired (contralesional) hemifield of vision. Once contrast thresholds had stabilized at normal levels of performance in each animal, DR and/or coherence thresholds were remeasured at the trained locations. $\boldsymbol{B}$, Effect of contrast sensitivity training on DR thresholds in cats with 17/18/19 lesions, showing a lack of transfer of learning. $\boldsymbol{C}$, Practicing the contrast sensitivity task also failed to improve DR thresholds in the impaired hemifield of cats with LS lesions. $\boldsymbol{D}$, Contrast sensitivity training also failed to transfer to coherence thresholds in cats with 17/18/19 lesions. $\boldsymbol{E}$, Practicing the contrast sensitivity task failed to improve coherence thresholds in the impaired hemifield of cats with LS lesions. Values are means \pm SEM; ${ }^{*} p<0.05$, two-tailed Student's $t$ test relative to intact hemifield performance (white bars). 0ther conventions are the same as those in Figure 6.

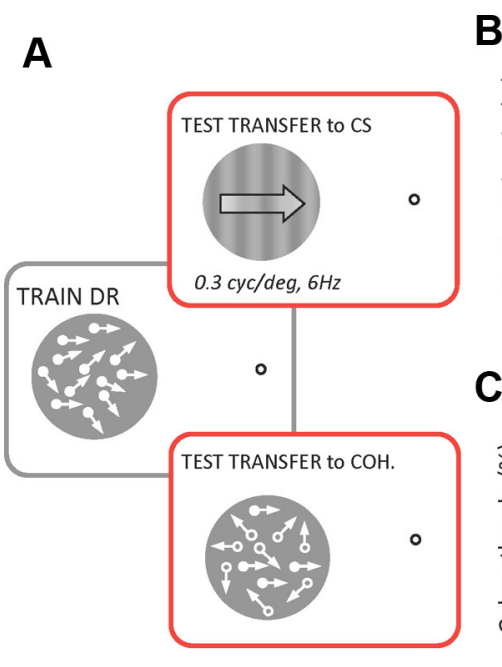

B $17 / 18 / 19$ lesions
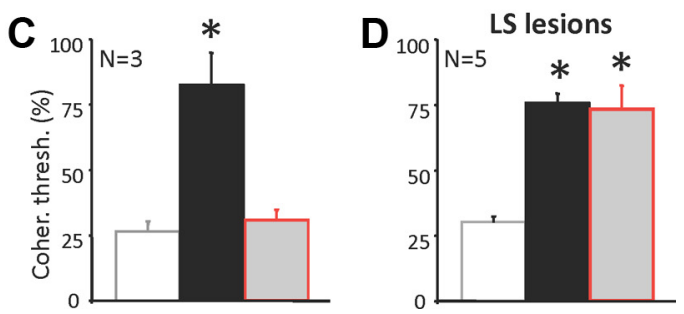

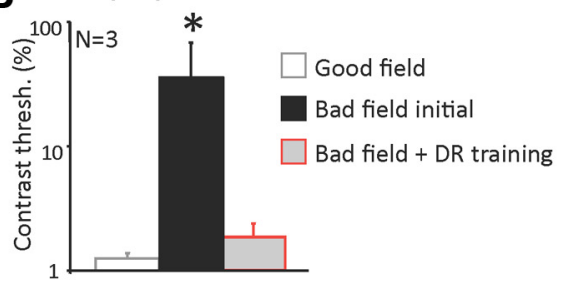

Figure 8. Global motion training improves contrast sensitivity for direction following lesions of areas $17 / 18 / 19$. $A$, Schematic diagram of experiment in which cats were trained to discriminate the left-right direction of motion of random dot stimuli in which the DR was varied from easier to harder levels in each session. After recovering normal DR thresholds, contrast thresholds were measured using drifting gratings and coherence thresholds were measured using random dot stimuli. $\boldsymbol{B}$, Plot of contrast thresholds in cats with 17/18/19 lesions either in the intact hemifield (white bar), the impaired hemifield before training (black bar), or at the same impaired hemifield locations after DR training (gray bar). DR training with random dot stimuli not only recovered DR thresholds (see Fig. 6) but also contrast sensitivity at trained locations in the impaired hemifield. C, Plot of coherence thresholds in cats with 17/18/19 lesions in the intact hemifield, the impaired hemifield before DR training, and after DR training, showing that DR training also improved coherence thresholds in these cats. This was in contrast with data obtained in five of the cats with $L S$ lesions and shown in $\boldsymbol{D}$, in which DR training did not improve coherence thresholds relative to pretraining levels. All values are means \pm SEM; ${ }^{*} p<0.05$, two-tailed Student's $t$ test relative to the intact hemifield values. Other conventions are the same as those in Figures 6 and 7. 

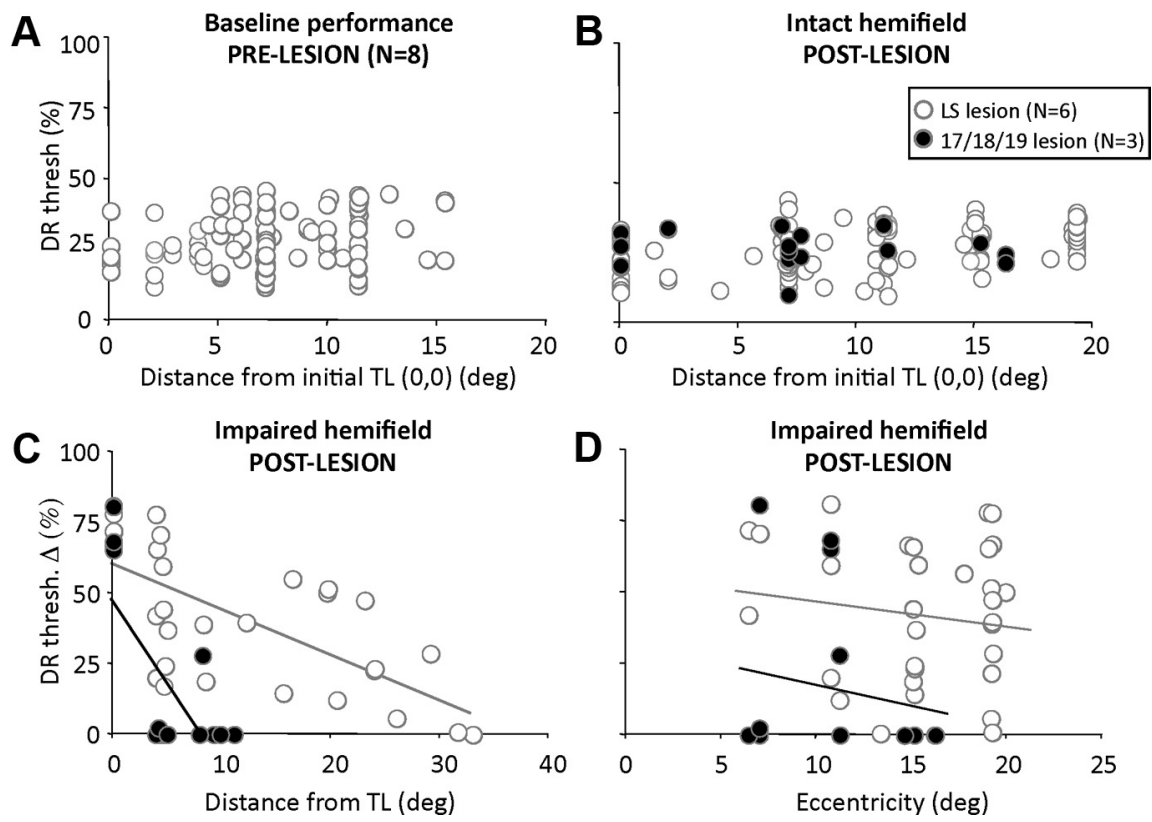

Figure 9. Retinotopic specificity of learning. $A$, Plot of DR thresholds in prelesion cats following initial behavioral training centrally. Once learning occurred and DR thresholds stabilized centrally, there was no significant drop in performance out to $\sim 16^{\circ}$ eccentricity in visually intact animals. Note that distance from trained location (TL) here is equivalent to eccentricity, with training location center coordinates $0,0 . \boldsymbol{B}$, Equivalent plot to that shown in $\boldsymbol{A}$, but with data collected postlesion in the center of the visual field $(0,0)$ and in the intact hemifield of six of the LS lesioned cats (white symbols) and all three of the cats with lesions of areas 17/18/19 (black symbols). Even postlesion, there was no significant correlation between DR thresholds and distance from the originally trained location at 0,0 in intact regions of the visual field. C, Retinotopic specificity of DR relearning postlesion in the same animals whose data are plotted in $\boldsymbol{B}$. Here, the magnitude of improvement in DR thresholds is plotted relative to pretraining (postlesion) values in cats with lesions of areas 17/18/19 (black dots) or LS cortex (white dots) as a function of distance from the trained (in this case, peripheral) impaired hemifield locations. Trained locations are always plotted at a distance of $0^{\circ}$. The magnitude of improvements decreased with increasing distance from the TLs. This decrease was significantly greater in cats with early visual cortex lesions than in cats with $L S$ lesions. $R^{2}=0.42$ for $L S$ lesions and 0.50 for $17 / 18 / 19$ lesions. $D$, Plot of change in DR thresholds relative to pretraining (postlesion) values versus eccentricity (degree), showing no significant correlation between training-induced improvement and eccentricity in either lesion group. $R^{2}=0.00002$ for LS lesions and 0.064 for $17 / 18 / 19$ lesions.
(Blackwell, 1946; Dean, 1981; Sclar et al., 1990). However, drifting gratings comprise a single motion vector that can be discriminated over a small region of the stimulus by lowlevel motion detectors (Reichardt, 1961; van Santen and Sperling, 1984; Adelson and Bergen, 1985; Watson and Ahumada, 1985; Lu and Sperling, 1995). On the other hand, accurate discrimination of global motion in a random dot stimulus requires detection followed by integration of multiple directional vectors (Braddick, 1974; Williams and Sekuler, 1984; Newsome and Paré, 1988; Watamaniuk et al., 1989; Nishida, 2011). When dots are sparse, as in our stimuli, and when there is a large range of dot directions or noise in the stimulus, integration must occur over a larger area of the stimulus to generate a reliable percept of net motion direction. We posit, therefore, that training with gratings may recruit and optimize function in fewer neurons, at lower levels of the visual cortical hierarchy, possibly with a narrower range of preferred directions, than random dot stimuli. When animals are subsequently exposed to random dot stimuli, this newly optimized circuitry may simply lack the spatial and/or directional bandwidth to compute global motion direction.

trast sensitivity for direction can also be recovered as a function of training. While pattern relearning following visual cortex damage in adult cats was previously reported (Spear and Braun, 1969; Wood et al., 1974; Spear and Baumann, 1979a,b), these studies differed from ours in that they used aspiration lesions, which damage both gray and white matter, and a static-grating-detection task, which did not measure contrast sensitivity per se.

Our results indicate much redundancy within the feline (and likely the primate) visual system with respect to processing of motion information. Such redundancy may explain why, following damage, intact visual areas, whether higher level (in the case of 17/18/19 lesions) or lower level (in the case of LS lesions), can relearn to process motion stimuli when appropriate training is provided. However, just because relearning occurs postlesion does not mean it is identical to learning with an intact visual system. An important question here deals with specificity to trained task and stimuli. Because the same task (left-right direction discrimination) was used for all testing and training conditions, we could examine specificity of learning for different stimulus attributes and observed the following:

1. Training to discriminate left-right directions of motion of low-spatial-frequency, low-contrast, drifting gratings did not improve discrimination of random dot stimuli, regardless of lesion location. Why? Both are first-order (luminance-defined) motion stimuli, where local luminance and contrast detection are the first processing step
2. In contrast, cats with $17 / 18 / 19$ lesions trained to extract global direction from random dot stimuli containing a large range of local directions (at least by the time training ended) exhibited improved contrast sensitivity for direction. This transfer is greatly reminiscent of that shown for humans with V1 lesions who trained similarly (Huxlin et al., 2009). One possible explanation for this result is that global motion processing requires local motion processing (and contrast detection) at its basis. Thus, if the subject can recover the ability to perform global motion discriminations, then, by inference, the subject must have recovered the ability to correctly detect local directions. An associated, training-induced improvement in signal-to-noise processing (DR training in these cats also improved coherence thresholds) may help explain the associated improvement in contrast sensitivity, although this remains speculative.

3. Finally, we observed a clear interaction between some of the training stimuli and lesion site: specifically, training on the DR task improved coherence thresholds in cats with 17/18/19 lesions, but not in cats with LS lesions (Huxlin and Pasternak, tex in cats and the MT area in primates are important sites of local motion integration (Movshon et al., 1985; Newsome and Paré, 1988; Pasternak and Merigan, 1994; Rudolph and Pasternak, 1996). The present data reveal an additional role of the feline LS cortex in generalization of learning between dif2004). Considerable evidence shows that such areas as LS cor- 
ferent global motion stimuli. The percept of global direction in DR stimuli results from pooling across detectors tuned to the mean direction of the local vector distribution (Williams and Sekuler, 1984). Pooling across a small numbers of these detectors (such as in strobe-reared cats) is sufficient to generate normal DR thresholds but not coherence thresholds (Pasternak et al., 1990). The latter presumably require pooling across a larger set of detectors or detectors with broader directional tuning (Britten and Newsome, 1998). Thus, when LS cortex is intact, cats can relearn and generalize DR learning to coherence. Without LS, if retraining with DR stimuli recruits a circuitry that pools across the smallest necessary range of local vectors to recover DR thresholds, this could be insufficient to attain normal coherence thresholds.

Overall, our data suggest that as long as an intact LS cortex is present, visual training on a global motion task can improve processing and sensitivity for multiple stimulus attributes. However, when LS cortex is damaged, the same training generalizes less. This is consistent with predictions of both the reverse hierarchy and rule-based learning theories: if learning occurs predominantly in higher-level areas, broader generalization across stimuli should result. Another consideration is that, following damage, some stimuli may more effectively recruit neurons in higher-level areas tied to effector/decision circuitries. In primates, motion-processing areas, such as the MT area, interact closely with effector/decision circuits in lateral intraparietal (LIP) (Roitman and Shadlen, 2002) and parietal cortex (Born and Bradley, 2005). Training monkeys to discriminate direction of motion of noisy stimuli (i.e., weak motion) improves sensory-driven responses in the LIP rather than the MT area (Law and Gold, 2008). Thus, following lesions, when basic discriminability of visual stimuli becomes more difficult, motion learning could result from improved readout and decision processes in higher-level areas rather than MT or LS cortex in cats. LS cortex projects to a higher-level, motion-sensitive region named the anterior ectosylvian visual area (AEV) (Olson and Graybiel, 1987; Scannell et al., 1996), an effector area linking sensory input with action (Jiang et al., 1994). Given AEV's known connections (Olson and Graybiel, 1987), its main direct source of visual information, if LS cortex is damaged, will come from the pulvinar/lateral posterior (LP) complex (AEV receives no direct projections from areas 17/18/19). When areas 17/ $18 / 19$ are damaged, visual information still reaches LS cortex (thence, AEV) via projections from the dorsal lateral geniculate nucleus (Sherk, 1986b; Tong and Spear, 1986) and the superior colliculus/LP complex/pulvinar (Kaufman et al., 1984). The spatiotemporal frequency content and preprocessing of visual information reaching AEV when areas 17/18/19 are damaged, may explain why motion relearning generalizes more broadly across stimulus attributes in these cats. The presence of tighter retinotopic organization in LS cortex (Grant and Shipp, 1991), as opposed to AEV (Olson and Graybiel, 1987; Scannell et al., 1996) and even LP/ pulvinar (Chalupa and Abramson, 1988), may also explain why relearning after early visual cortex lesions is retinotopically tighter to trained visual field locations than after LS lesions.

The tight spatial specificity of relearning in impaired hemifields we observed in cats with early visual cortex lesions also bears great similarity to the tight retinotopic specificity of relearning observed in the blind fields of V1-damaged humans (Sahraie et al., 2006; Huxlin et al., 2009). In both cases, retinotopic specificity of relearning contrasted with the generalizability of global motion discrimination performance across intact regions of the visual field, regardless of whether initial learning occurred before or after the lesion. These observations strongly suggest that retinotopic specificity of visual relearning may be a property of the cortical damage and the require- ment for directed training to elicit recovery at impaired sites, rather than a byproduct of the timing (prelesion or postlesion) of initial learning, or of the amount of training.

In summary, the hierarchical level of visual cortical damage does not appear to limit relearning of gross (left-right) motion discriminations in impaired portions of the visual field. However, both the locus of damage and the type of motion information in the retraining stimuli seemed to control generalizability of motion relearning. Much remains to be elucidated about the neural mechanisms underlying the significant training-induced plasticity displayed by damaged, adult visual systems. Defining the factors that control the effectiveness of training should both enhance our understanding of plasticity in the damaged brain, and help refine rehabilitation strategies for affected populations.

\section{References}

Adelson EH, Bergen JR (1985) Spatiotemporal energy models for the perception of motion. J Opt Soc Am A 2:284-299.

Ahissar M, Hochstein S (1997) Task difficulty and the specificity of perceptual learning. Nature 387:401-406.

Ahissar M, Hochstein S (2004) The reverse hierarchy theory of visual perceptual learning. Trends Cogn Sci 8:457-464.

Blackwell HR (1946) Contrast thresholds of the human eye. J Opt Soc Am 36:624-643.

Born RT, Bradley DC (2005) Structure and function of visual area MT. Ann Rev Neurosci 28:157-189.

Braddick O (1974) A short-range process in apparent motion. Vision Res 14:519-527.

Britten KH, Newsome WT (1998) Tuning bandwidths for near-threshold stimuli in area MT. J Neurophysiol 80:762-770.

Chalupa LM, Abramson BP (1988) Receptive field properties in the tectoand striate-recipient zones of the cat's lateral posterior nucleus. Prog Brain Res 75:85-94.

Dean AF (1981) The relationship between response amplitude and contrast for cat striate cortical neurones. J Physiol 318:413-427.

Dosher BA, Lu ZL (2009) Hebbian reweighting on stable representations in perceptual learning. Learn Percept 1:37-58.

Dosher BA, Lu ZL (1999) Mechanisms of perceptual learning. Vision Res 39:3197-3221.

Dreher B, Wang C, Turlejski KJ, Djavadian RL, Burke W (1996) Areas PMLS and 21a of cat visual cortex: two functionally distinct areas. Cereb Cortex 6:585-599.

Grant S, Shipp S (1991) Visuotopic organization of the lateral suprasylvian area and of an adjacent area of the ectosylvian gyrus of cat cortex: a physiological and connectional study. Vis Neurosci 6:315-338.

Hochstein S, Ahissar M (2002) View from the top: hierarchies and reverse hierarchies in the visual system. Neuron 36:791-804.

Huxlin KR, Pasternak T (2001) Long-term neurochemical changes after visual cortical lesions in the adult cat. J Comp Neurol 429:221-241.

Huxlin KR, Pasternak T (2004) Training-induced recovery of visual motion perception after extrastriate cortical damage in the adult cat. Cereb Cortex 14:81-90.

Huxlin KR, Williams JM, Price T (2008) A neurochemical signature of visual recovery after extrastriate cortical damage in the adult cat. J Comp Neurol 508:45-61.

Huxlin KR, Martin T, Kelly K, Riley M, Friedman DI, Burgin WS, Hayhoe M (2009) Perceptual re-learning of complex visual motion after V1 damage in humans. J Neurosci 29:3981-3991.

Jeter PE, Dosher BA, Petrov A, Lu ZL (2009) Task precision at transfer determines specificity of perceptual learning. J Vis 9:1, 1-13.

Jeter PE, Dosher BA, Liu SH, Lu ZL (2010) Specificity of perceptual learning increases with increased training. Vision Res 50:1928-1940.

Jiang H, Lepore F, Ptito M, Guillemot JP (1994) Sensory modality distribution in the anterior ectosylvian cortex (AEC) of cats. Exp Brain Res 97:404-414.

Karni A, Sagi D (1991) Where practice makes perfect in texture discrimination: evidence for primary visual cortex plasticity. Proc Natl Acad Sci U S A 88:4966-4970.

Kaufman EF, Rosenquist AC, Raczkowski D (1984) The projections of sin- 
gle thalamic neurons into multiple visual cortical areas in the cat. Brain Res 298:171-174.

Law CT, Gold JI (2008) Neural correlates of perceptual learning in a sensory-motor, but not a sensory, cortical area. Nat Neurosci 11:505-513.

Lee C, Weyand TG, Malpeli JG (1998) Thalamic control of cat lateral suprasylvian visual area-relation to patchy association projections from Area 18. Vis Neurosci 15:15-25.

Lu ZL, Sperling G (1995) The functional architecture of human visual motion perception. Vision Res 35:2697-2722.

Movshon JA, Adelson EH, Gizzi MS, Newsome WT (1985) The analysis of moving visual patterns. In: Pattern recognition mechanisms (Chagas C, Gattas R, Gross CG, eds), pp 117-151. Vatican City: Ponticifica Academia Scientiarum.

Newsome WT, Paré EB (1988) A selective impairment of motion perception following lesions of the middle temporal visual area (MT). J Neurosci 8:2201-2211.

Nishida S (2011) Advancement of motion psychophysics: review 20012010. J Vis 11:1-53.

Olson CR, Graybiel AM (1987) The ectosylvian visual area of the cat: location, retinotopic organization and connections. J Comp Neurol 261:277-294.

Palmer LA, Rosenquist AC, Tusa RJ (1978) The retinotopic organization of lateral suprasylvian visual areas in the cat. J Comp Neurol 177:237-256.

Pasternak T, Horn K (1991) Spatial vision of the cat: variation with eccentricity. Vis Neurosci 6:151-158.

Pasternak T, Maunsell JH (1992) Spatiotemporal sensitivity following lesions of area 18 in the cat. J Neurosci 12:4521-4529.

Pasternak T, Merigan WH (1994) Motion perception following lesions of the superior temporal sulcus in the monkey. Cereb Cortex 4:247-259.

Pasternak T, Horn KM, Maunsell JH (1989) Deficits in speed discrimination following lesions of the lateral suprasylvian cortex in the cat. Vis Neurosci 3:365-375.

Pasternak T, Albano JE, Harvitt DM (1990) The role of directionally selective neurons in the perception of global motion. J Neurosci 10:3079-3086.

Pasternak T, Tompkins J, Olson CR (1995) The role of striate cortex in visual function of the cat. J Neurosci 15:1940-1950.

Payne BR (1993) Evidence for visual cortical area homologs in cat and macaque monkey. Cereb Cortex 3:1-25.

Reichardt W (1961) Autocorrelation, a principle for the evaluation of sensory information by the central nervous system. In: Sensory communication (Rosenblith WA, ed), pp 303-317. New York: Wiley.

Roitman JD, Shadlen MN (2002) Response of neurons in the lateral intraparietal area during a combined visual discrimination reaction time task. J Neurosci 22:9475-9489.

Rudolph KK, Pasternak T (1996) Lesions in cat lateral suprasylvian cortex affect the perception of complex motion. Cereb Cortex 6:814-822.

Sagi D, Tanne D (1994) Perceptual learning: learning to see. Curr Opin Neurobiol 4:195-199.

Sahraie A, Trevethan CT, MacLeod MJ, Murray AD, Olson JA, Weiskrantz L (2006) Increased sensitivity after repeated stimulation of residual spatial channels in blindsight. Proc Natl Acad Sci U S A 103:14971-14976.

Scannell JW, Sengpiel F, Tovée MJ, Benson PJ, Blakemore C, Young MP (1996) Visual motion processing in the anterior ectosylvian sulcus of the cat. J Neurophysiol 76:895-907.

Sclar G, Maunsell JH, Lennie P (1990) Coding of image contrast in central visual pathways of the macaque monkey. Vision Res 30:1-10.
Sherk H (1986a) Location and connections of visual cortical areas in the cat's suprasylvian sulcus. J Comp Neurol 247:1-31.

Sherk H (1986b) Coincidence of patchy inputs from the lateral geniculate complex and area 17 to the cat's Clare-Bishop area. J Comp Neurol 253:105-120.

Sherk H, Mulligan KA (1993) A reassessment of the lower visual field map in striate-recipient lateral suprasylvian cortex. Vis Neurosci 10:131-158.

Spear PD, Baumann TP (1979a) Neurophysiological mechanisms of recovery from visual cortex damage in cats: properties of lateral suprasylvian visual area neurons following behavioural recovery. Exp Brain Res 35:177-192.

Spear PD, Baumann TP (1979b) Effects of visual cortex removal on receptive-field properties of neurons in lateral suprasylvian visual area of the cat. J Neurophysiol 42:31-56.

Spear PD, Braun JJ (1969) Pattern discrimination following removal of visual neocortex in the cat. Exp Neurol 25:331-348.

Symonds LL, Rosenquist AC (1984) Laminar origins of visual corticortical connections in the cat. J Comp Neurol 229:39-47.

Tong L, Spear PD (1986) Single thalamic neurons project to both lateral suprasylvian cortex and area 17: a retrograde fluorescent double labeling study. J Comp Neurol 246:254-264.

Tusa RJ, Palmer LA (1980) Retinotopic organization of areas 20 and 21 in the cat. J Comp Neurol 193:147-164.

Tusa RJ, Palmer LA, Rosenquist AC (1978) The retinotopic organization of area 17 (striate cortex) in the cat. J Comp Neurol 177:213-235.

Tusa RJ, Rosenquist AC, Palmer LA (1979) Retinotopic organization of areas 18 and 19 in the cat. J Comp Neurol 185:657-678.

Tusa RJ, Palmer LA, Rosenquist AC (1981) Multiple cortical visual areas. Visual field topography in the cat. In: Cortical sensory organisation (Woolsey CN, ed), pp 1-31. Clifton, NJ: Humana.

van Santen JP, Sperling G (1984) Temporal covariance model of human motion perception. J Opt Soc Am A 1:451-473.

Watamaniuk SN, Sekuler R, Williams DW (1989) Direction perception in complex dynamic displays: the integration of direction information. Vision Res 29:47-59.

Watson AB, Ahumada AJ Jr (1985) Model of human visual-motion sensing. J Opt Soc Am A 2:322-341.

Weibull W (1951) A statistical distribution function of wide applicability. J Appl Mech 18:292-297.

Williams DW, Sekuler R (1984) Coherent global motion percept from stochastic local motions. Vision Res 24:55-62.

Wood CC, Spear PD, Braun JJ (1974) Effects of sequential lesions of suprasylvian gyri and visual cortex on pattern discrimination in the cat. Brain Res 66:443-466.

Xiao LQ, Zhang JY, Wang R, Klein SA, Levi DM, Yu C (2008) Complete transfer of perceptual learning across retinal locations enabled by double training. Curr Biol 18:1922-1926.

Zhang X, Kedar S, Lynn MJ, Newman NJ, Biousse V (2006a) Natural history of homonymous hemianopia. Neurology 66:901-905.

Zhang X, Kedar S, Lynn MJ, Newman NJ, Biousse V (2006b) Homonymous hemianopias: clinical-anatomic correlations in 904 cases. Neurology 66:906-910.

Zhang JY, Zhang GL, Xiao LQ, Klein SA, Levi DM, Yu C (2010) Rule-based learning explains visual perceptual learning and its specificity and transfer. J Neurosci 30:12323-12328. 